\title{
Estado nutricional y condiciones de vida de los niños menores de cinco años del área urbana del municipio de Turbo, Antioquia, Colombia, 2004
}

\author{
Gloria Alcaraz ${ }^{1}$, Carlos Bernal ${ }^{2}$, William Cornejo ${ }^{3}$, Natalia Figueroa ${ }^{2}$, Margarita Múnera² \\ ${ }^{1}$ Facultad de Enfermería, Universidad de Antioquia, Medellín, Colombia \\ 2 Departamento de Pediatría y Puericultura, Universidad de Antioquia, Medellín, Colombia
}

Introducción. La desnutrición es un problema para quienes la padecen y para el futuro de las sociedades a las que pertenecen. Es importante conocer su magnitud para plantear soluciones. Objetivo. Evaluar el estado nutricional de los niños en el área urbana de Turbo por indicadores antropométricos y alimentarios, e identificar los factores sociodemográficos, ambientales, alimentarios y de salud asociados con el riesgo de desnutrición.

Materiales y métodos. Estudio descriptivo de corte transversal. La muestra estuvo conformada por 606 niños. Se hizo una selección aleatoria proporcional y polietápica de los barrios y manzanas del área urbana de Turbo. Se realizó evaluación antropométrica y aplicación de un cuestionario sobre aspectos socioeconómicos y antecedentes nutricionales a la madre.

Resultados. Utilizando un punto de corte de -2 unidades Z, las prevalencias de desnutrición global (insuficiencia ponderal), crónica y aguda fueron 11,1\% (IC95\% 8,5-13,6), 11,7\% (IC95\% $9,1-14,4)$ y $2,8 \%$ (IC95\% 1,4-4,2), respectivamente. Tomando como punto de corte -1 unidades Z, las prevalencias aumentaron a $41,7 \%$ (IC95\% 37,7-45,8), 36,9\% (IC95\% 33,0-40,9) y $24,6 \%$ (IC95\% 21,1-28,1), respectivamente. En el análisis bivariado se encontraron los mayores riesgos de desnutrición global, aguda y crónica para el sexo masculino; de desnutrición global y crónica, para mayores de un año; de desnutrición crónica, para niños que pertenecían a hogares de más de cinco miembros, hijos de mujeres con bajo nivel educativo, que recibieron lactancia materna por más de un año y que tenían más hermanos.

Conclusiones. La clasificación del estado nutricional utilizando indicadores antropométricos muestra que el riesgo de desnutrición en la población infantil del área urbana de Turbo estuvo por encima de los promedios nacionales. El estado nutricional está asociado con múltiples factores. De ahí, la importancia de abordarlos intersectorialmente para crear proyectos que den una respuesta integral a esta problemática.

Palabras clave: estado nutricional, trastornos de la nutrición del niño, condiciones sociales, indicadores de salud, lactancia materna, alimentación complementaria, políticas públicas de salud.

Nutritional status and living conditions in children in an urban area of Turbo, Antioquia, Colombia

Introduction. Malnutrition is a problem for the persons who suffer it and for the future of the societies to whom they belong. It is important to know its magnitude in order formulate solutions. Objective. The nutritional status of young children was evaluated in an urban area using anthropometric indices and factors associated with risk of malnutrition were identified-sociodemgraphic,environmental, nutritional, and health factors.

Materials and methods. In 2004, a cross-sectional descriptive survey was applied to 606 children, aged less than 5 years, in Turbo, a municipality in northwestern Colombia. A proportional, randomized, statistically significant sample of all the neighborhoods of the urban area in Turbo was used. Anthropometric evaluation (weight, height and age) was performed in all subjects, and a questionnaire regarding the socio-economical and nutritional background of the children was submitted to mothers and caregivers. 
Results. The prevalence percentages were as follows: low weight (11.1\%—Cl 8.5-13.6), stunting (11.7\%-Cl 9.1-14.4) and starvation (2.8\%- Cl 1.4-4.2). When a cut-off point of $-1 \mathrm{Z}$ score was applied, the prevalence increased to $41.7 \%(\mathrm{Cl} 37.7-45.8), 36.9 \%(\mathrm{Cl} 33.0-40.9)$ and $24.6 \%(\mathrm{Cl}$ 21.1-28.1) respectively. Bivariate analyses showed greater risks of global, severe and chronic malnutrition for males. Greater risk for global and chronic malnutrition occurred for children older than one year. Risk of chronic malnutrition was associated with the following conditions: children belonging to households with more than five people, children of mothers with low educational level, for children being breastfed for more than one year, and children who have a higher number of brothers.

Conclusions. An increased risk of malnutrition was present in the Turbo population when compared with the national anthropometric indices. The nutritional status of Turbo urban children is associated with several distinct socio-economical factors; these must be considered when implementing programs for the nutritional rehabilitation of this population.

Key words: nutritional status, child nutrition disorders, social conditions, health status indicators, breast feeding, supplementary feeding, health public policy

Según UNICEF, en el 2005, la pobreza fue la causa fundamental de las tasas elevadas de morbilidad y mortalidad en la infancia. Mil millones de niños de los países en desarrollo carecen, por lo menos, de uno de los bienes o servicios básicos que les permitirían sobrevivir, desarrollarse y prosperar; más de $16 \%$ de los menores de cinco años no reciben una nutrición adecuada y más de 16\% de los menores de cinco años de los países en desarrollo sufren desnutrición grave (1).

La desnutrición es uno de los problemas más serios en el mundo. Los niños son los más vulnerables debido a su rápido crecimiento y a su dependencia de otras personas. A pesar de los programas que se han implementado en el ámbito mundial para mejorar este problema, la alimentación es una de las necesidades básicas que permanece sin ser satisfecha entre los niños de todo el mundo.

La Encuesta Nacional de Demografía y Salud del 2000 mostró que $6,7 \%$ de los niños tenían insuficiencia ponderal (desnutrición global), 13,5\% talla corta (desnutrición crónica) y $0,8 \%$ emaciación (desnutrición aguda) (2). Según el informe mundial de UNICEF del 2002, la meta para

Correspondencia:

Gloria Alcaraz, Facultad de Enfermería, Universidad de Antioquia, Calle 64 № 53-09.

Teléfono: (574) 2106309 y 210 6308; fax: 2110058

glory@tone.udea.edu.co

Recibido: 28/05/07; aceptado: 16/10/07 el año 2000 era disminuir en $50 \%$ la desnutrición grave y moderada en los niños menores de cinco años de los países en desarrollo, pero sólo se logró en $17 \%$ (3). En Colombia, la meta era disminuirla en $50 \%$ y se alcanzó a disminuir en $43 \%$ (4).

Para el año 2005, según dicha encuesta, el 7\% de los niños colombianos presentaba insuficiencia ponderal (desnutrición global), 12\% talla corta (desnutrición crónica) y $1 \%$ emaciación (desnutrición aguda) (5). Sin embargo, es importante señalar que existen diferencias en los porcentajes de desnutrición según las regiones del país. Como se observa en las cifras generales de desnutrición, el problema en los niños colombianos continúa latente sin muchos cambios.

Para el departamento de Antioquia, la Encuesta Nacional de Demografía y Salud 2005 mostró prevalencias de insuficiencia ponderal (desnutrición global) de $6,1 \%$; de talla corta (desnutrición crónica) de $11,4 \%$ y de emaciación (desnutrición aguda) de $0,7 \%$ (5).

Un estudio realizado en la región de Urabá, donde está localizado el municipio de Turbo, mostró prevalencia de insuficiencia ponderal (desnutrición global) en $14,4 \%$, de talla corta (crónica) en 20,7\% y de emaciación (aguda) en 3,3\%, superando los índices departamentales y nacionales (6).

Existe un consenso general acerca de los múltiples factores que determinan la desnutrición. La inadecuada ingestión de alimentos y las enfermedades infecciosas son causas directas 
de este problema, pero hay factores sociodemográficos, ambientales, alimentarios, de salud y cuidado infantil, que de forma indirecta afectan el estado nutricional de los niños menores de cinco años $(1,5,7)$.

El objetivo de esta investigación fue conocer el estado nutricional de la población infantil de Turbo, con la utilización de indicadores antropométricos y alimentarios, e identificar la población en riesgo de desnutrición y los principales factores asociados con la desnutrición de estos niños, y aportar elementos que contribuyan a la formulación de proyectos para mejorar la situación nutricional de esta región.

\section{Materiales y métodos}

El estudio fue realizado en el municipio de Turbo, que hace parte de la región de Urabá y está ubicado al noroccidente de Colombia; tiene un área de $3.055 \mathrm{~km}^{2}$. La población estimada para el 2004 era de 123.604 habitantes, de los cuales, 39,7\% habitaban en el área urbana y, de éstos, el 17,1\% eran niños menores de cinco años (8).

Se realizó un estudio de tipo descriptivo, de corte transversal con asignación aleatoria, proporcional y polietápica, seleccionando al azar primero los barrios y, luego, las manzanas.

La muestra se calculó teniendo en cuenta la prevalencia estimada de desnutrición crónica en Colombia de 13,5\% para el año 2000 (2), una población de 9.799 niños menores de cinco años residentes en el área urbana de Turbo, según el censo de 1993 (9) y la Secretaría de Salud y Bienestar Social del municipio de Turbo, Antioquia, con un nivel de confianza de 95\%. Para calcular el tamaño de la muestra se utilizó el software Epiinfo versión 6.0 (Centers for Disease Control and Prevention, CDC, Atlanta), para un total de 540 niños menores de cinco años; sin embargo, como algunas manzanas tenían un elevado número de niños y se debían evaluar por pertenecer a la manzana seleccionada, el total de niños que ingresaron fue de 616 . Al depurar los registros antropométricos quedaron 606 niños.

Para garantizar la calidad de los datos, los investigadores fueron quienes recolectaron la información, previa estandarización de la toma de las medidas antropométricas y el diligenciamiento del cuestionario. Se realizó una prueba piloto con 20 niños seleccionados por conveniencia en dos de los barrios del área urbana del municipio, lo cual permitió detectar y corregir los problemas al diligenciar el cuestionario que se realizó a las madres o al acudiente de los niños.

Una auxiliar de enfermería ubicó las manzanas previamente seleccionadas, entregó una carta informativa a las madres o a las personas encargadas del cuidado de estos niños. Antes de la valoración nutricional, se solicitó la firma del consentimiento informado.

Se compararon los datos antropométricos con los valores de referencia del peso para la edad, la talla para la edad y el peso para la talla, del Nacional Center of Health Statistics/Organización Mundial de la Salud (NCHS/OMS), 1978 (10). Se calcularon las unidades $Z$ para cada indicador y se clasificaron como desnutridos los niños que estaban por debajo de -2 unidades $Z$ de la media de la población de referencia.

Para seleccionar la población infantil con mayor riesgo de desnutrición y sobre la cual se debe intervenir más oportunamente se utilizó, además, el punto de corte de -1 unidades $Z$ por debajo de la media de la población de referencia.

Para la toma del peso se retiraron los zapatos y la ropa; a todos los niños se les colocó una bata previamente estandarizada en su peso. Para los menores de dos años se utilizó una balanza mecánica Health o Meter ${ }^{\circledR}$, con sensibilidad de 1 $\mathrm{g}$ y capacidad de $65 \mathrm{~kg}$ y para los mayores de dos años se utilizó una balanza electrónica de pie marca LifeSource MD, modelo UC-321PX, con una sensibilidad de $0,05 \mathrm{~kg}$. La talla se tomó en decúbito supino en los menores de dos años con un infantómetro de madera con sensibilidad de 1 $\mathrm{mm}$. En los mayores de dos años la talla se tomó de pie con un estadiómetro marca Seca bodymeter 208 con sensibilidad de $1 \mathrm{~mm}$. Todos los niños se pesaron y midieron con el mismo equipo antropométrico. Cada medida (peso y talla) se tomó y registró dos veces; sólo se realizó una tercera toma si la diferencia entre las dos primeras sobrepasaba $10 \mathrm{~g}$, en el caso del peso o, $2 \mathrm{~mm}$, en el caso de la longitud. 
Se aplicó un cuestionario a la madre o a la persona que cuidaba al niño, en el cual se incluyeron preguntas para conocer las características sociodemográficas, económicas, alimentarias, ambientales y de salud de la población.

Se remitieron al hospital local aquellos niños que presentaron problemas de salud, y se hicieron conversatorios con las madres sobre el cuidado alimentario y nutricional de acuerdo con su situación económica y cultural.

Se diseñó una base de datos en el programa Excel $2003 \AA$ (Microsoft Corporation) y se exportó a Epiinfo 6.0 (CDC, Atlanta) y SPSS 11.5.0® para el análisis estadístico.

Con las variables categóricas se determinó la distribución de las frecuencias y, con las variables continuas, los valores máximos y mínimos, los valores promedio, las medidas de tendencia central y de dispersión, media, mediana y desviación estándar.

Se realizó un análisis exploratorio bivariado de cada una de las variables con respecto a la desnutrición global, crónica y aguda. La significancia estadística se determinó utilizando una prueba de ji al cuadrado de máxima verosimilitud del paquete estadístico Epiinfo 6.0 (CDC, Atlanta). La asociación se consideró significativa para niveles de probabilidad $p<0,05$. Se calcularon las razones de disparidad (odds ratio) con sus respectivos intervalos de confianza.

\section{Aspectos éticos}

El presente estudio se apoyó en los aspectos ético-legales considerados en la Resolución 008430 de 1993 del Ministerio de Salud de Colombia, referida a las normas científicas, técnicas y administrativas para la investigación en salud. Se tuvo en cuenta lo concerniente a los aspectos éticos de la investigación con seres humanos, la investigación en comunidades, la clasificación del riesgo y los compromisos que adquieren los investigadores (11). El proyecto fue aprobado por el Comité de Ética del Centro de Investigaciones de la Facultad de Medicina de la Universidad de Antioquia. Se obtuvo el consentimiento informado de todas las madres.

\section{Resultados}

\section{Características sociodemográficas}

Las características sociodemográficas de los 606 niños estudiados se encuentran en el cuadro 1; $47,5 \%$ eran niños y $52,5 \%$ niñas, con una edad promedio de 29,34 meses ( $D E=16,0$ ); $59,1 \%$ de los hogares estaban conformados por más de cinco personas y $80,2 \%$ de las familias tenía un ingreso mensual menor a un salario mínimo (el salario mínimo en Colombia en el 2004 fue de Col\$358.000). Los ingresos mensuales promedio eran de Col\$276.486 (equivalente a US $\$ 120$ o 92 euros).

El 3,2\% de las madres no tenía ningún tipo de educación y $35,2 \%$ terminaron la secundaria. De los niños estudiados, $32,8 \%$ eran hijos únicos y $45 \%$ eran los hijos menores (cuadro 1). El 93\% de la población consume agua de lluvia que almacena en tanques dentro de la vivienda, $77,5 \%$ de las viviendas poseen sanitario o letrina, las demás utilizan el campo abierto (cuadro 2).

\section{Características de nutrición y salud}

El 19,2\% de los niños nunca recibieron lactancia materna exclusiva, $71,9 \%$ la recibieron durante menos de tres meses y sólo $8,9 \%$ por más de seis meses. El promedio de lactancia materna total fue de 10 meses ( $D E=7,5$ ) (cuadro 3).

En el momento del estudio, $6,8 \%$ de los niños habían estado hospitalizados en los tres meses anteriores (cuadro 3); las causas fueron: asma, neumonía, impétigo, diarrea, fracturas, trauma craneoencefálico, intoxicación, convulsiones y sólo en dos casos la causa de la hospitalización fue por desnutrición aguda. El 57,1\% había estado enfermo en los 15 días previos, de los cuales, el $47,4 \%$ presentaron enfermedad respiratoria aguda; $5,8 \%$, enfermedad diarreica aguda, con una frecuencia baja para paludismo, otitis media aguda, fiebre, piodermitis, infección urinaria, conjuntivitis, asma, gingivitis, cefalea, neumonía y abscesos en piel (cuadro 3).

\section{Estado nutricional y su relación con las} características sociodemográficas y de salud

De los niños estudiados, se encontró insuficiencia ponderal (desnutrición global) en 11,1\% (IC95\%: 
Cuadro 1. Características sociodemográficas de los niños y de las familias de una muestra representativa de la población urbana de Turbo, Antioquia, Colombia, 2004.

\begin{tabular}{|c|c|c|c|}
\hline & & Frecuencia (n) & Porcentaje (\%) \\
\hline Edad (meses) & $\begin{array}{l}1-11 \\
12-23 \\
24-35 \\
36-47 \\
48-59\end{array}$ & $\begin{array}{l}112 \\
117 \\
142 \\
123 \\
112\end{array}$ & $\begin{array}{l}18,5 \\
19,3 \\
23,4 \\
20,3 \\
18,5\end{array}$ \\
\hline $\begin{array}{l}\text { Sexo } \\
\text { Tamaño familiar (personas) }\end{array}$ & $\begin{array}{l}\text { Masculino } \\
\text { Femenino } \\
<=5 \\
>5\end{array}$ & $\begin{array}{l}288 \\
318 \\
248 \\
358\end{array}$ & $\begin{array}{l}47,5 \\
52,5 \\
40,9 \\
59,1\end{array}$ \\
\hline Otros menores de 5 años en el hogar & $\begin{array}{l}0 \\
1 \\
2 \\
3 \\
4 \\
5\end{array}$ & $\begin{array}{r}273 \\
222 \\
72 \\
26 \\
11 \\
1\end{array}$ & $\begin{array}{r}45,1 \\
36,7 \\
11,9 \\
4,3 \\
1,8 \\
0,2\end{array}$ \\
\hline Hijo menor & $\begin{array}{l}\text { Sí } \\
\text { No }\end{array}$ & $\begin{array}{l}273 \\
128\end{array}$ & $\begin{array}{l}45,1 \\
21,2\end{array}$ \\
\hline Hijo único & $\begin{array}{l}\text { Sí } \\
\text { No }\end{array}$ & $\begin{array}{l}199 \\
407\end{array}$ & $\begin{array}{l}32,8 \\
67,2\end{array}$ \\
\hline Edad materna al momento del nacimiento del niño (años) & $\begin{array}{l}<18 \\
18-25 \\
26-35 \\
>35\end{array}$ & $\begin{array}{r}82 \\
323 \\
143 \\
50\end{array}$ & $\begin{array}{l}13,7 \\
54 \\
23,9 \\
8,4\end{array}$ \\
\hline Educación materna & $\begin{array}{l}\text { Ninguna } \\
\text { Primaria incompleta } \\
\text { Primaria completa } \\
\text { Secundaria incompleta } \\
\text { Secundaria completa } \\
\text { Universidad } \\
\text { Técnico }\end{array}$ & $\begin{array}{r}19 \\
68 \\
79 \\
165 \\
212 \\
30 \\
30\end{array}$ & $\begin{array}{r}3,2 \\
11,3 \\
13,1 \\
27,4 \\
35,2 \\
5 \\
5\end{array}$ \\
\hline Estado civil de la madre & $\begin{array}{l}\text { Soltera } \\
\text { Casada } \\
\text { Unión libre } \\
\text { Viuda } \\
\text { Separada }\end{array}$ & $\begin{array}{r}171 \\
67 \\
346 \\
4 \\
18\end{array}$ & $\begin{array}{c}28,2 \\
11,1 \\
57,1 \\
0,7 \\
3\end{array}$ \\
\hline Controles prenatales & $\begin{array}{l}<4 \\
>=4\end{array}$ & $\begin{array}{l}137 \\
459\end{array}$ & $\begin{array}{l}23 \\
77\end{array}$ \\
\hline Intervalo de nacimiento (meses) & $\begin{array}{l}<24 \\
24 \text { a } 48 \\
>48\end{array}$ & $\begin{array}{r}54 \\
184 \\
110\end{array}$ & $\begin{array}{l}15,5 \\
52,9 \\
31,6\end{array}$ \\
\hline $\begin{array}{l}\text { Ingresos mensuales por familia }{ }^{1} \\
\text { (salarios mínimos) }\end{array}$ & $\begin{array}{l}<1 \\
1 \text { a } 2 \\
>2\end{array}$ & $\begin{array}{r}481 \\
93 \\
26\end{array}$ & $\begin{array}{r}80,2 \\
15,5 \\
4,3\end{array}$ \\
\hline Desplazados & $\begin{array}{l}\text { Sí } \\
\text { No }\end{array}$ & $\begin{array}{r}70 \\
536\end{array}$ & $\begin{array}{l}11,6 \\
88,4\end{array}$ \\
\hline
\end{tabular}

${ }^{1}$ Salario mínimo en Colombia (2004) \$358.000 
Cuadro 2. Indicadores de saneamiento de los hogares de los niños de una muestra representativa de la población urbana de Turbo, Antioquia, Colombia, 2004.

\begin{tabular}{llcc}
\hline & & Frecuencia (n) & Porcentaje (\%) \\
\hline \multirow{2}{*}{ Agua de consumo diario } & Acueducto & 259 & 42,9 \\
& Pozo & 24 & 4 \\
& Agua lluvia & 565 & 93,2 \\
& Bolsa & 8 & 1,3 \\
Disposición de excretas & Sanitario & 409 & 67,7 \\
& Letrina & 59 & 9,8 \\
Disposición de basuras & Campo abierto & 105 & 17,4 \\
& Pozo séptico & 38 & 6,3 \\
& Relleno sanitario & 18 & 3 \\
& Quema & 50 & 8,3 \\
& Carro de basura & 432 & 71,5 \\
\hline
\end{tabular}

Cuadro 3. Indicadores de salud estudiados en los niños de una muestra representativa de la población urbana de Turbo, Antioquia, Colombia, 2004

\begin{tabular}{|c|c|c|c|}
\hline & & Frecuencia (n) & Porcentaje (\%) \\
\hline Lactancia materna actual & $\begin{array}{l}\text { Sí } \\
\text { No }\end{array}$ & $\begin{array}{l}125 \\
478\end{array}$ & $\begin{array}{l}20,7 \\
79,3\end{array}$ \\
\hline Tiempo total de lactancia materna (meses) & $\begin{array}{l}0 \text { a } 6 \\
7 \text { a } 12 \\
13 \text { a } 24 \\
>=25\end{array}$ & $\begin{array}{r}171 \\
192 \\
95 \\
15\end{array}$ & $\begin{array}{r}29,1 \\
32,7 \\
16,2 \\
2,6\end{array}$ \\
\hline Lactancia materna exclusiva (meses) & $\begin{array}{l}<2 \\
2-3 \\
4 \text { a } 6 \\
>6\end{array}$ & $\begin{array}{r}310 \\
103 \\
112 \\
51\end{array}$ & $\begin{array}{r}53,8 \\
17,9 \\
19,4 \\
8,9\end{array}$ \\
\hline Inicio de la alimentación complementaria (meses) & $\begin{array}{l}0 \text { a } 3 \\
4 \text { a } 6 \\
7 \text { a } 12 \\
>=13\end{array}$ & $\begin{array}{r}219 \\
270 \\
76 \\
6\end{array}$ & $\begin{array}{c}36,9 \\
45,5 \\
12,8 \\
1\end{array}$ \\
\hline Enfermedad en los últimos 15 días & $\begin{array}{l}\text { Sí } \\
\text { No }\end{array}$ & $\begin{array}{l}346 \\
258\end{array}$ & $\begin{array}{l}57,3 \\
42,7\end{array}$ \\
\hline Diarrea & $\begin{array}{l}\text { Sí } \\
\text { No }\end{array}$ & $\begin{array}{r}35 \\
569\end{array}$ & $\begin{array}{r}5,8 \\
94,2\end{array}$ \\
\hline Infección respiratoria aguda & $\begin{array}{l}\text { Sí } \\
\text { No }\end{array}$ & $\begin{array}{l}286 \\
318\end{array}$ & $\begin{array}{l}47,4 \\
52,6\end{array}$ \\
\hline Hospitalización en los últimos 3 meses & $\begin{array}{l}\mathrm{Si} \\
\text { No }\end{array}$ & $\begin{array}{r}41 \\
563\end{array}$ & $\begin{array}{r}6,8 \\
93,2\end{array}$ \\
\hline Edemas & $\begin{array}{l}\mathrm{Si} \\
\text { No }\end{array}$ & $\begin{array}{r}0 \\
606\end{array}$ & $\begin{array}{r}0 \\
100\end{array}$ \\
\hline
\end{tabular}


$8,5-13,6)$, talla corta (desnutrición crónica) en 11,7\% (IC95\%: 9,1-14,4) y emaciación (desnutrición aguda) en 2,8\% (IC95\%: 1,4-4,2). El promedio del puntaje Z para el indicador peso/ edad fue de -0,69; para talla/edad fue de $-0,70$ y el de peso/talla fue de -0,33.

Sin embargo, al tomar el punto de corte de -1 unidades $Z$ por debajo de la media de la población de referencia, para detectar a los niños con riesgo de desnutrición, se encontró que 41,7\% (IC95\%: 37,7-45,8) de los niños presentaban insuficiencia ponderal, 36,9\% (IC95\%: 33,0-40,9) talla corta y 24,6\% (IC95\%:21,1-28,1) emaciación.
Los cuadros 4 y 5 muestran la distribución de las frecuencias de desnutrición según las características sociodemográficas de la población y según indicadores de salud y nutrición.

Los hombres tuvieron mayor riesgo de insuficiencia ponderal, talla corta y emaciación para cada uno de los indicadores $(p<0,001)$. Los niños de 12 a 23 meses y de 36 a 59 meses mostraron mayores prevalencias de insuficiencia ponderal y de talla corta $(p=0,001)$ (cuadro 6). Presentaron mayor riesgo de talla corta los niños que vivían en familias de más de cinco personas $(p=0,013)$. Los hijos de madres con educación universitaria o

Cuadro 4. Distribución de la frecuencia de desnutrición según las características sociodemográficas de los niños de una muestra representativa de la población urbana de Turbo, Antioquia, Colombia, 2004.

\begin{tabular}{|c|c|c|c|c|}
\hline Variable & frec & $\begin{array}{l}\text { Aguda } \\
n=145 \\
\text { uencia (\%) }\end{array}$ & $\begin{array}{c}\text { Global } \\
n=250 \\
\text { frecuencia (\%) }\end{array}$ & $\begin{array}{c}\text { Crónica } \\
n=221 \\
\text { frecuencia (\%) }\end{array}$ \\
\hline \multicolumn{5}{|l|}{ Sexo } \\
\hline & Masculino & $108(74,5)$ & $173(69,2)$ & $127(57,4)$ \\
\hline & Femenino & $37(25,5)$ & $77(30,8)$ & $94(42,5)$ \\
\hline \multicolumn{5}{|c|}{ Edad (meses) } \\
\hline & $1-11$ & $22(15,1)$ & $29(11,6)$ & $23(10,4)$ \\
\hline & $12-23$ & $36(24,8)$ & $54(21,6)$ & $51(23,0)$ \\
\hline & $24-35$ & $30(20,6)$ & $53(21,2)$ & $43(19,4)$ \\
\hline & $36-47$ & $31(21,3)$ & $58(23,2)$ & $49(22,1)$ \\
\hline & $48-59$ & $26(17,9)$ & $56(22,4)$ & $55(24,8)$ \\
\hline \multicolumn{5}{|l|}{ Hijo único } \\
\hline & Sí & $40(27,5)$ & $67(26,8)$ & $57(25,7)$ \\
\hline & No & $105(72,4)$ & $183(73,2)$ & $164(74,2)$ \\
\hline \multicolumn{5}{|c|}{ Hijo menor ${ }^{2}$} \\
\hline & Sí & $74(70,4)$ & $129(71,6)$ & $112(69,1)$ \\
\hline & No & $31(29,5)$ & $51(28,3)$ & $50(30,8)$ \\
\hline \multicolumn{5}{|c|}{ Escolaridad } \\
\hline & Ninguna & $2(1,3)$ & $8(3,2)$ & $9(4,0)$ \\
\hline & Primaria incompleta & $16(11,0)$ & $32(12,8)$ & $30(13,5)$ \\
\hline & Primaria completa & $23(15,8)$ & $43(17,2)$ & $37(16,7)$ \\
\hline & Secundaria incompleta & $44(30,3)$ & $77(30,8)$ & $65(29,4)$ \\
\hline & Secundaria completa & $48(33,1)$ & $74(29,6)$ & $72(32,5)$ \\
\hline & Universidad & $7(4,8)$ & $10(4,0)$ & $5(2,2)$ \\
\hline & Técnico & $5(3,4)$ & $6(2,4)$ & $3(1,3)$ \\
\hline \multicolumn{5}{|c|}{ Ingresos mensuales por familia } \\
\hline & Menos de un salario mínimo 3 & $107(73,7)$ & $197(78,8)$ & $192(86,8)$ \\
\hline & 1-2 salarios mínimos & $28(19,3)$ & $39(15,6)$ & $21(9,5)$ \\
\hline & Más de 2 salarios mínimos & $10(6,8)$ & $14(5,6)$ & $8(3,6)$ \\
\hline \multicolumn{5}{|c|}{ Tamaño familiar } \\
\hline & Menor o igual a 5 personas & $52(35,8)$ & $91(36,4)$ & $76(34,3)$ \\
\hline & Más de 5 personas & $93(64,1)$ & $159(63,6)$ & $145(65,6)$ \\
\hline
\end{tabular}

\footnotetext{
${ }^{1}$ Hijo menor excluyendo los hijos únicos

${ }^{2}$ Salario mínimo en Colombia (2004) \$358.000
} 
Cuadro 5. Distribución de la frecuencia de desnutrición en relación con los indicadores de salud y nutrición de los niños de una muestra representativa de la población urbana de Turbo, Antioquia, Colombia, 2004.

\begin{tabular}{|c|c|c|c|}
\hline Variable & $\begin{array}{c}\text { Aguda } \\
n=145 \\
\text { frecuencia (\%) }\end{array}$ & $\begin{array}{c}\text { Global } \\
n=250 \\
\text { frecuencia (\%) }\end{array}$ & $\begin{array}{c}\text { Crónica } \\
n=221 \\
\text { frecuencia (\%) }\end{array}$ \\
\hline \multicolumn{4}{|l|}{ Lactancia materna actual } \\
\hline Sí & $28(19,3)$ & $36(14,4)$ & $35(15,8)$ \\
\hline No & $117(80,6)$ & $214(85,6)$ & $186(84,1)$ \\
\hline \multicolumn{4}{|c|}{ Tiempo total de lactancia materna (meses) ${ }^{4}$} \\
\hline $0-6$ & $34(29,8)$ & $66(31,7)$ & $57(31,1)$ \\
\hline $7-12$ & $47(41,2)$ & $80(38,4)$ & $66(36,0)$ \\
\hline $13-24$ & $27(23,6)$ & $51(24,5)$ & $51(27,8)$ \\
\hline$>=25$ & $6(5,2)$ & $11(5,2)$ & $9(4,9)$ \\
\hline \multicolumn{4}{|c|}{ Inicio de alimentación complementaria (meses) ${ }^{5}$} \\
\hline $0-3$ & $47(33,3)$ & $77(32,3)$ & $83(39,9)$ \\
\hline $4-6$ & $70(49,6)$ & $116(48,7)$ & $89(42,7)$ \\
\hline $7-12$ & $21(14,8)$ & $42(17,6)$ & $33(15,8)$ \\
\hline$>=13$ & $3(2,1)$ & $3(1,2)$ & $3(1,4)$ \\
\hline \multicolumn{4}{|c|}{ Hospitalizado en los últimos 3 meses } \\
\hline Sí & $9(6,2)$ & $17(6,8)$ & $21(9,5)$ \\
\hline No & $136(93,7)$ & $233(93,2)$ & $200(90,4)$ \\
\hline \multicolumn{4}{|l|}{ Enfermos en los últimos 15 días } \\
\hline Sí & $88(60,6)$ & $143(57,2)$ & $123(55,6)$ \\
\hline No & $57(39,3)$ & $107(42,8)$ & $98(44,3)$ \\
\hline
\end{tabular}

${ }^{1}$ Excluyendo los niños que estaban recibiendo lactancia materna

${ }^{2}$ Excluyendo los niños que no habían iniciado alimentación complementaria

técnica y los hijos únicos tuvieron menor riesgo de insuficiencia ponderal y talla corta, en forma significativa $(p<0,001)$ (cuadro 6).

Se encontró, según la estadística, mayor riesgo de insuficiencia ponderal y talla corta en niños entre los mayores de seis meses que recibían lactancia materna (cuadro 6).

\section{Discusión}

La desnutrición en los niños del municipio de Turbo, utilizando como punto de corte - 2 unidades $Z$, en relación con el patrón de referencia, es más elevada que los promedios nacionales para desnutrición global y aguda.

Sin embargo, al tomar como punto de corte -1 unidad $Z$ con respecto al patrón de referencia, el porcentaje de riesgo de desnutrición se eleva en forma notable a $41,9 \%$ para la insuficiencia ponderal, $36,9 \%$ para la talla corta y $24,0 \%$ para la emaciación o delgadez. Esto significa que una proporción muy importante de los niños se encuentra entre -1 y -2 unidades $Z$ : $30 \%$ para insuficiencia ponderal, 25,3\% para desnutrición talla corta y $21,8 \%$ para emaciación o delgadez.

El punto de corte de -2 unidades $Z$ es el aceptado internacionalmente; sin embargo, sólo detecta a los niños con riesgo de desnutrición moderada y grave. Si se acepta como punto de corte -1 unidad $Z$ y se considera con riesgo de desnutrición a los niños que se encuentran entre -1 y -2 unidades $Z$, se detectan en forma oportuna los niños con riesgo de desnutrición. El disminuir el punto de corte a 1 unidad $Z$ aumenta la sensibilidad para detectar a los niños con desnutrición, aun cuando disminuye la especificidad, porque algunos niños que están entre -1 y -2 unidades $Z$ no son realmente desnutridos sino niños delgados o con talla relativamente baja.

El proceso de desnutrición es silencioso y los niños con desnutrición leve están a un paso de la desnutrición moderada y, éstos, a un paso para llegar a la desnutrición grave con peligro inminente de muerte. Pelletier señala que el riesgo de muerte infantil es más elevado en los niños con desnutrición leve y moderada en relación con los 
Cuadro 6. Análisis bivariado de los factores de riesgo de desnutrición aguda, global y crónica de los niños de la población urbana de Turbo, Antioquia, Colombia, 2004.

\begin{tabular}{|c|c|c|c|c|c|c|c|c|c|}
\hline \multirow[t]{2}{*}{ Variable } & \multicolumn{3}{|c|}{ Desnutrición aguda } & \multicolumn{3}{|c|}{ Desnutrición global } & \multicolumn{3}{|c|}{ Desnutrición crónica } \\
\hline & & $\mathbf{R}^{*}$ (IC 95\%) & p & OR & (IC 95\%) & p & & (IC 95\%) & p \\
\hline \multicolumn{10}{|l|}{ Sexo } \\
\hline Masculino & & 1 & & & 1 & & & 1 & \\
\hline Femenino & 0,22 & $(0,14-0,34)$ & $<0,001$ & 0,21 & $(0,15-0,31)$ & $<0,001$ & 0,53 & $(0,38-0,75)$ & $<0,001$ \\
\hline \multicolumn{10}{|l|}{ Edad (meses) } \\
\hline $1-11$ & & 1 & & & 1 & & & 1 & \\
\hline $12-23$ & 1,82 & $(0,95-3,50)$ & 0,052 & 2,45 & $(1,35-4,46)$ & 1 & 2,99 & $(1,60-5,61)$ & $<0,001$ \\
\hline $24-35$ & 1,10 & $(0,57-2,12$ & 0,77 & 1,70 & $(0,96-3,04)$ & 0,053 & 1,68 & $(0,90-3,14)$ & 0,078 \\
\hline $36-47$ & 1,38 & $(0,71-2,68)$ & 0,30 & 2,55 & $(1,42-4,61)$ & $<0,001$ & 2,56 & $(1,38-4,79)$ & 0,001 \\
\hline $48-59$ & 1,24 & $(0,62-2,46)$ & 0,51 & 2,86 & $(1,57-5,23)$ & $<0,001$ & 3,73 & $(1,99-7,04)$ & $<0,001$ \\
\hline \multicolumn{10}{|l|}{ Hijo único } \\
\hline Sí & & 1 & & & 1 & & & 1 & \\
\hline No & 1,38 & $(0,90-2,13)$ & 0,12 & 1,61 & $(1,11-2,33)$ & 0,007 & 1,68 & $(1,15-2,47)$ & 0,005 \\
\hline \multicolumn{10}{|l|}{ Hijo menor } \\
\hline Sí & & 1 & & & 1 & & & 1 & \\
\hline No & 0,86 & $(0,51-1,43)$ & 0,53 & 0,74 & $(0,47-1,16)$ & 0,16 & 0,92 & $(0,59-1,45)$ & 0,70 \\
\hline \multicolumn{10}{|l|}{ Escolaridad } \\
\hline Superior & & 1 & & & 1 & & & 1 & \\
\hline Secundaria & 1,29 & $(0,63-2,69)$ & 0,45 & 1,84 & $(0,96-3,54)$ & 0,047 & 3,71 & $(1,64-8,72)$ & $<0,001$ \\
\hline Primaria & 1,31 & $(0,60-2,89)$ & 0,46 & 2,75 & $(1,38-5,54)$ & 0,001 & 5,49 & $(2,33-13,38)$ & $<0,001$ \\
\hline \multicolumn{10}{|l|}{$\begin{array}{l}\text { Ingresos mensuales } \\
\text { por familia }\end{array}$} \\
\hline$<1$ salario mínimo & & 1 & & & 1 & & & 1 & \\
\hline 1-2 salarios mínimos & 1,51 & $(0,89-2,53)$ & 0,10 & 1,04 & $(0,65-1,67)$ & 0,86 & 0,44 & $(0,25-0,76)$ & 0,001 \\
\hline >2 salarios mínimos & 2,18 & $(0,89-5,28)$ & 0,55 & 1,68 & $(0,72-3,97)$ & 0,19 & 0,67 & $(0,26-1,67)$ & 0,35 \\
\hline \multicolumn{10}{|l|}{ Tamaño familiar } \\
\hline$<=$ a 5 personas & & 1 & & & 1 & & & 1 & \\
\hline$>5$ personas & 1,32 & $(0,88-1,98)$ & 0,15 & 1,38 & $(0,98-1,95)$ & 0,057 & 1,64 & $(1,08-2,20)$ & 0,013 \\
\hline \multicolumn{10}{|c|}{ Lactancia materna actual } \\
\hline No & 1,12 & $(0,69-1,85)$ & 0,62 & 2,00 & $(1,28-3,14)$ & 0,001 & 1,64 & $(1,04-2,58)$ & 0,024 \\
\hline \multicolumn{10}{|l|}{$\begin{array}{l}\text { Tiempo total lactancia } \\
\text { materna (meses) }\end{array}$} \\
\hline $0-6$ & & 1 & & & 1 & & & 1 & \\
\hline $7-12$ & 1,31 & $(0,77-2,22)$ & 0,29 & 1,14 & $(0,73-1,77)$ & 0,55 & 1,05 & $(0,66-1,66)$ & 0,83 \\
\hline $13-24$ & 1,60 & $(0,86-2,98)$ & 0,11 & 1,84 & $(1,08-3,16)$ & 0,017 & 2,32 & $(1,34-4,01)$ & 0,001 \\
\hline$>=25$ & 2,69 & $(0,78-9,02)$ & 0,069 & 4,38 & $(1,22-17,09)$ & 0,008 & 3,00 & $(0,92-10,06)$ & 0,038 \\
\hline \multicolumn{10}{|l|}{$\begin{array}{l}\text { Inicio alimentación } \\
\text { complementaria (meses) }\end{array}$} \\
\hline $0-3$ & & 1 & & & 1 & & & 1 & 0,25 \\
\hline $4-6$ & 1,28 & $(0,82-2,00)$ & 0,25 & 1,39 & $(0,95-2,04)$ & 0,079 & 0,81 & $(0,55-1,19)$ & 0,39 \\
\hline $7-12$ & 1,40 & $(0,74-2,64)$ & 0,27 & 2,28 & $(1,30-4,01)$ & 0,002 & 1,26 & $(0,72-2,21)$ & 0,54 \\
\hline$>=13$ & 3,66 & $(0,57-23,65)$ & 0,097 & 1,84 & $(0,29-11,77)$ & 0,45 & 1,64 & $(0,26-10,45)$ & \\
\hline \multicolumn{10}{|c|}{ Enfermo en los últimos 15 días } \\
\hline Sí & & 1 & & & 1 & & & 1 & \\
\hline No & 0,83 & $(0,56-1,24)$ & 0,34 & 1,01 & $(0,72-1,41)$ & 0,97 & 1,05 & $(0,74-1,49)$ & 0,76 \\
\hline \multicolumn{10}{|l|}{$\begin{array}{l}\text { Hospitalizado en los últimos } \\
3 \text { meses }\end{array}$} \\
\hline Sí & & 1 & & & 1 & & & 1 & \\
\hline No & 1,13 & $(0,50-2,62)$ & 0,74 & 1,00 & $(0,50-1,99)$ & 0,99 & 0,52 & $(0,27-1,04)$ & 0,043 \\
\hline
\end{tabular}

* OR: odds ratio: razón de disparidad; IC95\%: intervalo de confianza 95\% 
eutróficos $(12,13)$. El utilizar solamente el punto de corte de -2 unidades $Z$, enmascara la presencia de desnutrición leve en los niños y proponemos utilizar el puntaje $Z$ de -1 para detectar e intervenir oportunamente el problema de la desnutrición en la población infantil.

En la mayoría de los estudios, el sexo no ha sido identificado como un factor de riesgo para la desnutrición crónica (7,14-16); sin embargo, en un estudio se encontró mayor riesgo de este tipo de desnutrición en el sexo femenino (17), datos que contrastan con lo demostrado en la presente investigación: mayor riesgo de desnutrición en los niños que en las niñas. No obstante, en las entrevistas y en los conversatorios con las madres sobre salud y alimentación, no se encontraron indicios sobre la preferencia en el cuidado de las niñas frente a los niños.

El mayor riesgo de talla corta en los niños colombianos se presenta entre los 12 y 23 meses de edad (5), a diferencia de lo encontrado en Turbo, en donde el mayor riesgo de insuficiencia ponderal y talla corta, ocurre entre los 48 y 59 meses.

En los niños del estudio se encontró que la prevalencia de talla corta aumenta a partir del año de edad. Según De Onis (18), el cuerpo de un niño reacciona a la mala nutrición de diversas maneras que se pueden medir por medio de la antropometría: pérdida de peso como reacción a una baja ingestión de alimentos, o desaceleración o suspensión del crecimiento que, a largo plazo, se refleja en la baja estatura para la edad. La inseguridad alimentaria que viven muchos niños de Turbo (19) y las precarias condiciones económicas y ambientales van condicionando el incremento de la talla corta en la población infantil a medida que aumenta la edad.

Entre los factores socioeconómicos que influyen en el estado nutricional de los niños están el pertenecer a una familia con más de cinco personas y el número de niños menores de cinco años por familia $(17,20)$. Según la Encuesta Nacional de Demografía y Salud 2005 (5), los niños que pertenecen a familias con 6 o más hijos presentan mayor porcentaje de insuficiencia ponderal y talla corta. También se ha encontrado asociación entre ser hijo único y un menor riesgo de desnutrición $(14,17)$. En el trabajo de campo, se observó que, cuando existen varios niños en un mismo hogar, las madres deben repartir su tiempo para el cuidado de cada uno de ellos y, en ocasiones, no alcanzan a suplir sus necesidades. A medida que aumenta el número de miembros en una familia, también se debe incrementar la disponibilidad de recursos para adquirir alimentos. En la mayoría de los casos esto no se logra y, por lo tanto, se pone en riesgo el estado nutricional de todos los miembros de la familia y, especialmente, el de los menores de cinco años.

El mayor nivel de escolaridad de la madre repercute favorablemente en el estado nutricional de los niños, tal como se señala en la literatura $(5,14)$ y se encontró en esta investigación. No obstante, si el alto nivel de escolaridad de las madres de Turbo se acompañara de una mejor oferta laboral, seguramente las cifras de desnutrición infantil caerían y se podría asegurar un futuro más exitoso para esta población.

A pesar de que el Plan Nacional de Alimentación y Nutrición, 1996-2006, de Colombia (21), así como los organismos internacionales, promueven la lactancia materna exclusiva durante los primeros seis meses de vida de los niños, esta meta en nuestro medio está lejos de cumplirse. En Turbo se observa que el tiempo de lactancia materna exclusiva no cubre los plazos recomendados por la OMS y el inicio de la alimentación complementaria se da antes de los seis meses de edad. Existe una presión familiar y social grande para que los niños inicien tempranamente el consumo de leche entera de vaca y de biberones preparados con plátano (Musa paradisiaca), alimentos que no cubren las necesidades de macro nutrientes y micronutrientes que tienen los niños a estas edades (19). Es escaso el número de madres que suministran a sus hijos fórmulas infantiles adaptadas a la edad de los niños, bien por problemas económicos para su compra o por razones culturales (19).

Según la Encuesta Nacional de Demografía y Salud 2005, el valor de la mediana en el tiempo de lactancia materna exclusiva es 3,7 meses y la mediana en tiempo de lactancia materna es de 16,3 meses (5). En contraste, el presente estudio 
en Turbo muestra que $53,8 \%$ recibieron lactancia materna exclusiva menos de dos meses y $71,9 \%$ recibieron lactancia materna durante menos de tres meses.

La asociación estadística encontrada en este estudio, entre la lactancia materna después del año de vida y la desnutrición global y crónica, podría explicarse por las condiciones desfavorables del medio ambiente y por la alimentación complementaria deficiente en estos niños, lo cual sería finalmente lo que provocaría la desnutrición por no tener un buen balance de nutrientes, ni las necesidades calóricas suficientes para el crecimiento de los niños. La lactancia materna acompañada de una alimentación complementaria adecuada sería una forma eficaz para asegurar el estado nutricional de los niños de Turbo.

Los factores asociados con el riesgo de desnutrición en los menores de cinco años de este municipio son: ser hombre, ser mayor de un año, pertenecer a familia numerosa y ser hijo de madre con baja escolaridad. En este estudio no se encontró asociación entre ingresos económicos y desnutrición. Llama la atención que la emaciación y la insuficiencia ponderal fueron ligeramente superiores en los niños de familias con mayores ingresos económicos, aun cuando no en forma significativa; esto puede explicarse porque sólo 26 niños pertenecían a familias con ingresos superiores a dos salarios mínimos y a la posible interacción con otras variables, como tamaño de la familia, escolaridad de las madres y aspectos culturales, entre otras.

A pesar de que la desnutrición, generalmente, está asociada con la pobreza y con la presencia de enfermedades infecciosas, otros factores, como el medio ambiente, la cultura y la comunicación en salud, son también factores determinantes. Son necesarios programas claros, coherentes y adaptados a la cultura sobre lactancia materna, alimentación complementaria y cuidado de la salud de la madre y el niño. Se requiere la participación de cada colectivo en la formulación de programas para asegurar la apropiación y la sustentabilidad en las prácticas de alimentación.

Los datos aportados por el estudio pueden permitir a las autoridades del departamento de Antioquia, del municipio de Turbo y al colectivo turbeño, estructurar programas para mejorar las condiciones de vida y salud de la población infantil y, por ende, contribuir al desarrollo futuro del municipio.

\section{Conflicto de intereses}

Los autores manifestamos que no existe ningún conflicto de interés.

\section{Financiación}

La investigación fue financiada por la Universidad de Antioquia y por la Dirección Local de Salud de Turbo.

\section{Referencias}

1. UNICEF. Estado Mundial de la Infancia, 2005. La infancia amenazada. Nueva York: Fondo de las Naciones Unidas para la Infancia; 2004. p.10-30.

2. Ojeda G, Ordóñez M, Ochoa L. Salud sexual y reproductiva en Colombia. Encuesta Nacional de Demografía y Salud. Bogotá: Profamilia; 2000. p.145-50.

3. UNICEF. Estado mundial de la infancia 2002. La infancia y el desarrollo en el decenio de 1990. Nueva York: UNICEF; 2002. p.16-7.

4. UNICEF Colombia. Estado de la infancia colombiana. Cumbre mundial a favor de la infancia. 2002. [Consultado: 24 de noviembre de 2006]. Disponible en: http://www.unicef.org.co/pdf/estado-colombia.pdf

5. Ojeda G, Ordóñez M, Ochoa L. Salud sexual y reproductiva en Colombia. Encuesta Nacional de Demografía y Salud. Bogotá: Profamilia; 2005. p.252$5 ; 267-71$.

6. Álvarez MC, Benjumea MV, Roldán $\mathbf{P}$, Maya $\mathbf{M}$, Montoya EC. Perfil alimentario y nutricional de los hogares de la región del Bajo Cauca antioqueño. Medellín: Gobernación de Antioquia, Dirección Seccional de Salud, Programa de Mejoramiento Alimentario y Nutricional de Antioquia, Universidad de Antioquia, Escuela de Nutrición y Dietética; 2005.

7. Abate G, Kogi-Makau W, Muroki NM. Child-feeding practices as predictors of nutritional status of children in slum area in Addis Ababa, Ethiopia. Ethiop J Health Dev. 1999;13:229-38.

8. Dirección Seccional de Salud de Antioquia. Población 2002. Medellín: DSSA; 2002. [Consultado: 13 de marzo de 2002]. Disponible en: www.dssa. gov.co/htm/población.htm

9. Departamento Administrativo Nacional de Estadística DANE. Censo 1993. Santa Fe de Bogotá: DANE; 2000. [Consultado: 3 de marzo de 2002]. Disponible en: http://www.dane.gov.co/files/ investigaciones/poblacion/poblacion_vivienda/ poblacion_colombia.XLS 
10. Hamil PVV, Drizid TA, Johnson CL, Reed RB, Roche AF, Moore WM. Physical growth: National Center for Health Satistics percentiles. Am J Clin Nutr. 1979;32:607-29.

11. Londoño JL, Alvarado EJ, Casas JV, Roselli DA. Normas científicas, técnicas y administrativas para la investigación en salud. Resolución № 008430 de 1993. Por la cual se establecen las normas científicas, técnicas y administrativas para la investigación en salud. Santa Fe de Bogotá: Ministerio de Salud, Dirección de Desarrollo Científico y Tecnológico; 1993. p.2-8.

12. Pelletier DL. The relationship between child anthropometry and mortality in developing countries: Implications for policy programs and future research. J Nutr 1994;124:2047S-81S.

13. Pelletier DL, Frongillo EA Jr, Habicht JP. Epidemiologic evidence for a potentiating effect of malnutrition on child mortality. Am J Public Health. 1993;83:1130-3.

14. Yimer G. Malnutrition among children in Southern Ethiopia: Levels and risk factors. Ethiop J Health Dev. 2000;14:283-92.

15. Hernández-Martínez E, Roldán-Fernández SG. Prevalencia de desnutrición en preescolares de Tabasco, México. Salud Pública Mex. 1995;37:211-8.

16. Dodero E, Falcón A, Paz FM, Rodríguez R, Rodríguez LM. Frecuencia de desnutrición en niños que asisten a centros de aprendizaje para preescolares. Rev Mex Pediatr. 2001;68:132-4.

17. Instituto APOYO. Política Nacional para la Reducción de la Desnutrición Crónica en el Perú. Revisión de la literatura nacional e internacional referida a factores asociados a desnutrición crónica. Una aproximación desde la metodología del meta-análisis [Documento electrónico]. Lima: Instituto APOYO; 2001. [Consultado: 30 de noviembre de 2006]. Disponible en: http:// www.prisma.org.pe/download/Estudio Complementario.pdf

18. De Onis M. Medición del estado nutricional con relación a la mortalidad. Bol Med Hosp Infant Mex. 2002;59:70-5.

19. Alcaraz GM, Bernal C, Aristizábal MA, Ruíz MB, Fox JE. Anemia y anemia por déficit de hierro en niños menores de cinco años y su relación con el consumo de hierro en la alimentación. Turbo, Antioquia, Colombia. Invest Educ Enferm. 2006;24:16-29.

20. Kariuki FN, Monari JM, Kibui MM, Mwirichia MA, Zani KK, Tetei M, et al. Prevalence and risk factors of malnutrition. J Natl Inst Public Health. 2002;51:44-50.

21. Departamento Nacional de Planeación, Consejería para la Política Social. Plan Nacional de Alimentación y Nutrición 1996-2005. Bogotá: Departamento Nacional de Planeación y Consejería para la Política Social; 1996. p.118. 